\title{
Multilevel Associations with Cancer Screening Among Women in Rural, Segregated Communities Within the Northeastern USA: a Mixed-Methods Study
}

\author{
Jennifer L. Moss ${ }^{1}$ (D) $\cdot$ Kelsey Leach ${ }^{1} \cdot$ Kelsey C. Stoltzfus ${ }^{1} \cdot$ Marni Granzow $^{1} \cdot$ Paul L. Reiter ${ }^{2} \cdot$ Tracy Onega $^{3,4}$. \\ Lisa M. Klesges ${ }^{5} \cdot$ Mack T. Ruffin IV $^{1}$
}

Accepted: 5 July 2021 / Published online: 15 July 2021

(c) American Association for Cancer Education 2021

\begin{abstract}
We recruited women (primarily non-Hispanic White) from 14 rural, segregated counties in a Northeastern US state for an explanatory sequential study: 100 women (ages 50-65 years) completed a survey, and 16 women participated in focus groups. We sought to identify personal (e.g., healthcare mistrust) and environmental (e.g., travel time to healthcare providers) factors related to colorectal and cervical cancer screening. Quantitatively, $89 \%$ of participants were up-to-date for cervical screening, and $65 \%$ for colorectal screening. Factors interacted such that compounding barriers were associated with lower odds of screening (e.g., insurance status and healthcare mistrust: interaction $p=.02$ for cervical; interaction $p=.05$ for colorectal). Qualitatively, three themes emerged regarding barriers to screening: privacy concerns, logistical barriers, and lack of trust in adequacy of healthcare services. While cancer screening was common in rural, segregated counties, women who reported both environmental and personal barriers to screening had lower uptake. Future interventions to promote screening can target these barriers.
\end{abstract}

Keywords Cancer screening $\cdot$ Cervical cancer $\cdot$ Colorectal cancer $\cdot$ Rural $\cdot$ Racial residential segregation $\cdot$ Women's health

More than 4000 women die from cervical cancer, and more than 20,000 women die from colorectal cancer, each year in the USA [1]. However, deaths from these cancers could be prevented through routine use of screening tests available in primary care settings: Pap tests and/or human papillomavirus (HPV) test for cervical cancer [2] and visualization and/or stool tests for colorectal cancer [3]. Routine cancer

Jennifer L. Moss

jmoss1@pennstatehealth.psu.edu

1 Department of Family and Community Medicine, Department of Public Health Sciences, Penn State College of Medicine, The Pennsylvania State University, 134 Sipe Ave., \#205, MC HS72, P.O. Box 850, Hershey, PA 17033, USA

2 College of Public Health, The Ohio State University, Columbus, OH, USA

3 Huntsman Cancer Institute, Salt Lake City, UT, USA

4 University of Utah, Salt Lake City, UT, USA

5 Washington University School of Medicine in St. Louis, St. Louis, MO, USA screening allows for early detection of cancer, which can lead to a better prognosis [2,3]. Screening for cervical and colorectal cancers, however, is characterized by several health disparities, including by rurality $[4,5]$ and by racial residential segregation [6]. Outcomes for these (and other screen-detectable) cancers are correspondingly poorer in rural $[7,8]$ and segregated $[9,10]$ counties than in other parts of the USA.

Multilevel social determinants may explain how these screening disparities have emerged [11]. Adults in vulnerable communities (including rural and segregated counties) face multilevel barriers to healthcare, including personal factors (e.g., low healthcare trust [12], high cancer fatalism [13]) and environmental factors (e.g., long travel time to a clinic [14], high area-level poverty [15]). Multilevel frameworks, such as social cognitive theory [16] and Warnecke's [17] model for population health, suggest that personal and environmental factors operate independently and interactively in their relationships with health outcomes. However, we have limited understanding of how these multilevel factors characterize the cancer screening behaviors of women living in rural and racially segregated communities. 
This mixed-methods study aims to assess multilevel factors related to cancer screening among women living in rural, segregated counties in Pennsylvania to support future interventions. For the quantitative portion of the study, we hypothesized that survey data would indicate that being up-to-date with cervical and colorectal cancer screening is lower among women reporting more barriers to screening. For the qualitative portion of the study, we identified themes that elaborated on the social, personal, and physical barriers to screening. These findings can inform multilevel interventions and implementation strategies to reduce barriers to screening, increase screening behaviors, and reduce cancer burden in vulnerable communities.

\section{Materials and Methods}

\section{Procedures}

We recruited women ages 50-65 (i.e., typically eligible for routine screening for cervical [2] and colorectal [3] cancers according to US Preventive Services Task Force (USPSTF) guidelines in effect in June, 2019) from community settings in 14 eligible counties in Pennsylvania for a mixed-methods, explanatory sequential study. Eligible counties were (1) nonmetropolitan according to the 2010 United States Department of Agriculture [18] rural-urban continuum codes, and (2) above the national median for the dissimilarity index [19] assessing residential segregation for non-Hispanic whites versus all others in census tracts within counties, using the 2010 United States Census Bureau data [20].

Recruitment modalities included study flyers posted in central locations, in-person contact at clinics and community events, and social media posts about the study. Potential participants completed a brief eligibility screening by paper, phone, or Internet. Study inclusion criteria were (1) female gender, (2) age 50-65 years, (3) living in one of the eligible counties, and (4) able to complete a survey or participate in a focus group in English.

For the quantitative phase, participants provided implied consent before completing the survey, which was either administered by an interviewer (in-person or by telephone) or self-administered online. Data collection took place between June 2019, and April 2020. The survey included 53 questions and took an average of $18 \mathrm{~min}$ to complete (including eligibility screening, consent procedures, and survey administration). At the conclusion of the survey, participants received a copy of the consent form, a thank you note, and a \$10 gift card (physical or electronic versions were available). In total, we screened 150 women, 35 of whom were ineligible (24/35 were ineligible due to living outside of the eligible counties). Of the remaining 115 eligible women, 15 did not complete the survey; all of these non-completers accessed the survey online. Thus, the final analytic sample included 100 participants.

For the qualitative phase, we held three focus groups with survey participants $(n=16)$. Data collection took place in May 2020. We invited participants who had completed the entire survey and who gave permission for us to re-contact them; we contacted participants until we finished scheduling all three focus groups (total $n$ approached=21). Prior to the focus group, participants provided verbal consent for participation. These focus groups were held virtually because of physical distancing policies as a result of the COVID-19 pandemic, using a teleconferencing platform to enable audio and video interaction. A female, masters'-level study coordinator (KS) and two female note-takers (including JM, KL, MG) managed the focus groups using a semi-structured discussion guide (Supplementary information file S1) designed to elicit opinions about factors related to cancer screening. The data collection team had previously engaged in training on conduct of focus groups, and the team held a mock focus group to pilot-test procedures before beginning data collection. On average, focus groups lasted 61 min (including a brief orientation to the teleconferencing platform, an ice breaker, and discussion guide questions). Focus groups were audio-recorded and transcribed. At the conclusion of the focus group, participants received a $\$ 40$ electronic gift card sent to their e-mail address.

Representativeness The survey $(n=100)$ and focus group $(n=16)$ participants were fairly representative of the general population in the 14 eligible counties from which they were drawn (Table 1). For example, according to data from the 2015-2019 American Community Survey [21], among the entire population in the counties, $71 \%$ had private health insurance, while this percentage was $68 \%$ in survey participants and $75 \%$ in focus group participants. However, the participants were less representative of the population in terms of race/ethnicity ( $92 \%$ of population in non-Hispanic white, compared to $99 \%$ of survey participants and $100 \%$ of focus group participants) and educational attainment (42\% of population has greater than a high school degree, compared to $72 \%$ of survey participants and $75 \%$ of focus group participants).

\section{Measures}

Quantitative Phase We assessed whether participants were up-to-date for cervical cancer screening [2] if they (1) had a Pap test in the last 3 years and/or (2) had an HPV test in the last 5 years. We assessed whether participants were upto-date for colorectal cancer screening [3] if they (1) had a visualization test (colonoscopy or sigmoidoscopy) in the last 5 years or (2) had a blood stool test in the last year (defined to be consistent with NCI Small Area Estimates). We also 
Table 1 Descriptive statistics for sample of women (ages 50-65) in rural, segregated counties of Pennsylvania, 2019-2020 ( $n=100$ for surveys; $n=16$ for focus groups)

\begin{tabular}{|c|c|c|c|c|}
\hline & \multicolumn{2}{|c|}{ Survey participants } & \multicolumn{2}{|c|}{$\begin{array}{l}\text { Focus group par- } \\
\text { ticipants }\end{array}$} \\
\hline & Mean & $S E$ & Mean & $S E$ \\
\hline Age, range: $50-65$ years & 57.3 & 0.4 & 58.8 & 1.2 \\
\hline \multirow[t]{2}{*}{ Self-rated health, range: 1 (poor)-5 (excellent) } & 3.5 & 0.1 & 3.6 & 0.2 \\
\hline & $n$ & $\%$ & $n$ & $\%$ \\
\hline \multicolumn{5}{|l|}{ Race/ethnicity } \\
\hline Non-Hispanic white & 99 & 99.0 & 16 & 100.0 \\
\hline Other & 1 & 1.0 & 0 & 0.0 \\
\hline \multicolumn{5}{|l|}{ Annual household income } \\
\hline$<\$ 50,000$ & 45 & 48.9 & 4 & 26.7 \\
\hline$\$ 50,000$ or more & 47 & 51.1 & 11 & 73.3 \\
\hline \multicolumn{5}{|l|}{ Educational attainment } \\
\hline High school degree or less & 28 & 28.0 & 4 & 25.0 \\
\hline More than high school degree & 72 & 72.0 & 12 & 75.0 \\
\hline \multicolumn{5}{|l|}{ Marital status } \\
\hline Not married/living with a partner & 36 & 36.4 & 4 & 25.0 \\
\hline Married/living with a partner & 63 & 63.6 & 12 & 75.0 \\
\hline \multicolumn{5}{|l|}{ Insurance status } \\
\hline Non-private & 32 & 32.0 & 4 & 25.0 \\
\hline Private & 68 & 68.0 & 12 & 75.0 \\
\hline \multicolumn{5}{|l|}{ Check-up in last year } \\
\hline No & 17 & 17.0 & 3 & 18.8 \\
\hline Yes & 83 & 83.0 & 13 & 81.3 \\
\hline \multicolumn{5}{|l|}{ Had a hysterectomy } \\
\hline No & 64 & 64.0 & 9 & 56.3 \\
\hline Yes & 36 & 36.0 & 7 & 43.8 \\
\hline
\end{tabular}

created a composite variable indicating if participants were up-to-date on both cervical and colorectal cancer screening.

We measured four constructs of personal factors related to cancer screening: healthcare trust, health self-efficacy, cancer worry, and cancer fatalism (see complete items in Supplementary Table S1). We measured healthcare trust with three items [22]; items were analyzed individually given the low internal consistency of the scale (Cronbach's $\alpha=0.25$ ). Health self-efficacy and cancer worry were measured with a single item each [23]. We measured cancer fatalism with three items [23]; items were analyzed individually given the low internal consistency of the scale (Cronbach's $\alpha=0.61$ ).

We also created two indices of barriers to screening for each type of cancer, adapted from Janz et al. [24]. Separately for cervical and colorectal cancers, we asked participants about common barriers to screening (do not know how; do not need to; embarrassed; afraid; cost is too high; doctor is too far away) (Supplementary Table S1). We summed the number of barriers to screening for each cancer endorsed by each participant to create an index (potential range: 0-6).

For environmental factors related to screening, we measured travel time by asking participants how long (in minutes) it takes them to travel from their home to their doctor's office
(Supplementary Table S1). We also measured perceived community social cohesion with two items adapted from O'Brien et al. [25], with higher scores indicating greater social cohesion; we averaged participant responses to these two items.

Control variables include self-reported participant age (in years), self-rated health, annual household income, educational attainment, marital status, insurance status, check-up in the last year, and history of hysterectomy (Supplementary Table S1).

Qualitative Phase The focus group discussion guide included items and probes assessing social, personal, and physical factors related to cancer screening. Example items include "What are the barriers you face in getting tested for cancer?" and "How might these be different for you compared to women who live in different communities?" Data collection continued until thematic saturation was achieved, as assessed by saturation tables [26]. Specifically, we assessed when each theme was first discussed by focus group members and evaluated whether subsequent focus groups introduced novel concepts. 


\section{Analysis}

Quantitative Phase Descriptive statistics summarized the control variables and multilevel factors. Bivariate logistic regressions and chi-square tests assessed differences in cancer screening behaviors across levels of the control variables. For multivariable analysis, we retained control variables that were associated with either cancer screening outcome in preliminary bivariate analysis ( $p<0.05$; data not shown): annual household income, insurance status, check-up in the last year, and (for cervical cancer screening) history of hysterectomy.

Logistic regression modeling examined the relationships between the personal and environmental factors and being up-to-date with each screening, adjusting for the control variables. We assessed potential moderation by creating multiplicative interaction terms for each personal factor with travel time (i.e., cross-level interaction) or insurance status. We limited the candidate moderators to reduce the threat of multiple comparison error; these two candidate moderators were selected based on theoretical relevance to access to primary care. When Wald chi-square statistics indicated that the interaction term contributed significantly $(p<0.10)$ to explaining the outcome variable, we probed the interaction by stratifying across levels of travel time or insurance status (as applicable).

Analyses were conducted using SAS version 9.4 (Cary, NC). The primary statistical tests used a two-sided $p$-value of 0.05 . Exploratory statistical tests (i.e., moderation analysis) used a two-sided $p$-value of 0.10 .

Qualitative Phase We used content analysis [27, 28] to explore multilevel factors related to cancer screening using transcripts from the focus groups. The codebook included a "start list" [27, 29] of codes developed from the discussion guide, supplemented by emergent themes identified in the data. At least two study team members independently coded each transcript via the nVivo software (Burlington, MA), and discrepancies were resolved through discussion until consensus was reached. Below, we highlight themes that elaborate on the quantitative findings, following an explanatory sequential mixed-methods study design.

The Pennsylvania State University Human Subjects Protections Office approved data collection and analysis for this project.

\section{Results}

\section{Findings from Quantitative Survey Phase}

On average, participants were 57.3 years old (standard error $(S E)=0.4)($ Table 1$)$. About half $(51.1 \%)$ of participants lived in households with an annual income of $\$ 50,000$ or more, and most had private insurance $(68.0 \%)$ and had had a check-up in the last year $(83.0 \%)$.

Participants had moderate levels of healthcare trust (i.e., mean scores of $2.2(S E=0.1)$ to $3.6(S E=0.1))$ and low-to-moderate levels of cancer fatalism (i.e., mean scores of $1.9(S E=0.1)$ to $2.6(S E=0.1))($ Table 2). Out of 6 possible barriers, participants endorsed $0.8(S E=0.1)$ barriers to cervical cancer screening and $1.2(S E=0.1)$ barriers to colorectal cancer screening. The most common barriers to cancer screening were embarrassment (cervical: 24\%; colorectal: 38\%), cost (cervical: 19\%; colorectal: $27 \%$ ), and fear (cervical: $14 \%$; colorectal: $25 \%$ ). On average, participants traveled $20.5(S E=1.4)$ min from their home to their doctor's office (Table 2).

Main Effects Overall, $88.9 \%$ of participants were up-to-date with cervical cancer screening (excluding women who had a hysterectomy, $85.9 \%$ were up-to-date), and $65.0 \%$ were upto-date with colorectal cancer screening. As a result, $60.0 \%$ were up-to-date with screenings for both cancers (excluding women who had a hysterectomy, $50.0 \%$ were up-to-date with screenings for both cancers). Adjusting for control variables, none of the personal or environmental factors was associated with screening (all $p>0.05$ ) (Table 3 ).

Interaction Effects Travel time moderated the relationship between one of the cancer fatalism items, it seems like everything causes cancer, and (1) cervical cancer screening (interaction $p=0.10$, Fig. 1A) and (2) screening for both cancers (interaction $p=0.01$, Fig. 1B). Specifically, cancer fatalism was not associated with screening for participants with low ( $>1$ standard deviation below the mean) or moderate (between \pm 1 standard deviation around the mean) travel times, but fatalism was negatively associated with screening among participants with high ( $>1$ standard deviation above the mean) travel times. As an illustrative comparison, among participants with high travel times, uptake of cervical cancer screening decreased from $98 \%$ among participants who somewhat disagreed that it seems like everything causes cancer, to $90 \%$ among participants who somewhat agreed, to $56 \%$ among participants who strongly agreed; participants with low or moderate travel times demonstrated no relationship between fatalism and screening (Fig. 1A).

In addition, insurance status moderated the relationship between several personal factors and screening (for cervical cancer: healthcare trust, cancer worry; for colorectal cancer: healthcare trust, cancer fatalism; for both cancers: healthcare trust, cancer fatalism). For example, healthcare trust (agreeing that patients should follow medical advice) was positively associated with screening for participants who were privately insured, but negatively associated with screening for participants who were not privately insured (cervical: 
Table 2 Personal and environmental factors related to cervical and colorectal cancer screening among women (ages 50-65) in rural, segregated counties of Pennsylvania, 2019-2020 $(n=100)$

\begin{tabular}{|c|c|c|}
\hline & Mean & $S E$ \\
\hline \multicolumn{3}{|l|}{ Personal factors } \\
\hline \multicolumn{3}{|l|}{ Healthcare trust, each range: 1 (low trust)-4 (high trust) } \\
\hline Healthcare more concerned about making money (rev) & 2.2 & 0.1 \\
\hline Patients should follow medical advice & 3.0 & 0.1 \\
\hline Would see a doctor for medical problem & 3.6 & 0.1 \\
\hline General health self-efficacy, range: 1 (low)-5 (high) & 4.0 & 0.1 \\
\hline Cancer worry, range: 1 (low)-5 (high) & 2.6 & 0.1 \\
\hline \multicolumn{3}{|l|}{ Cancer fatalism, each range: 1 (low)-4 (high) } \\
\hline It seems like everything causes cancer & 2.6 & 0.1 \\
\hline There's not much to do to lower cancer chances & 1.9 & 0.1 \\
\hline Hard to know which cancer prevention recommendations to follow & 2.9 & 0.1 \\
\hline \multicolumn{3}{|l|}{ Number of barriers to screening ${ }^{1}$, range: $0-6$} \\
\hline Cervical cancer & 0.8 & 0.1 \\
\hline Colorectal cancer & 1.2 & 0.1 \\
\hline \multicolumn{3}{|l|}{ Environmental factors } \\
\hline Travel time to doctor's office, range: $1.5-60 \mathrm{~min}$ & 20.5 & 1.4 \\
\hline Low travel time ( $>1$ standard deviation below mean) & 4.6 & 0.3 \\
\hline Medium travel time (between \pm 1 standard deviation around mean) & 18.3 & 0.9 \\
\hline High travel time ( $>1$ standard deviation above mean) & 45.9 & 1.7 \\
\hline Community social cohesion, range: 1 (low) -4 (high) & 3.3 & 0.1 \\
\hline
\end{tabular}

${ }^{1}$ Barriers for screening for each cancer included do not know how to; do not need to; embarrassed; afraid; cost is too high; doctor is too far away (Supplementary Table S1)

Table 3 Associations between personal and environmental factors related to cervical and colorectal cancer screening among women (ages 50-65) in rural, segregated counties of Pennsylvania, 2019-2020 $(n=100)$

\begin{tabular}{|c|c|c|c|c|c|c|}
\hline & \multicolumn{2}{|c|}{ Cervical cancer } & \multicolumn{2}{|c|}{ Colorectal cancer } & \multicolumn{2}{|c|}{ Both cancers } \\
\hline & OR & $95 \% \mathrm{CI}$ & OR & $95 \% \mathrm{CI}$ & OR & $95 \% \mathrm{CI}$ \\
\hline \multicolumn{7}{|l|}{ Personal factors } \\
\hline \multicolumn{7}{|l|}{ Healthcare trust } \\
\hline Healthcare more concerned about making money (rev) & 1.52 & $(0.59-3.93)$ & 1.04 & $(0.58-1.87)$ & 0.88 & $(0.44-1.75)$ \\
\hline Patients should follow medical advice & 1.07 & $(0.29-3.93)$ & 1.46 & $(0.71-3.01)$ & 0.90 & $(0.37-2.21)$ \\
\hline Would see a doctor for medical problem & 0.96 & $(0.36-2.57)$ & 1.36 & $(0.73-2.53)$ & 1.11 & $(0.54-2.31)$ \\
\hline General health self-efficacy & 0.79 & $(0.26-2.34)$ & 0.86 & $(0.44-1.66)$ & 0.66 & $(0.30-1.45)$ \\
\hline Cancer worry & 2.01 & $(0.81-4.95)$ & 1.03 & $(0.68-1.56)$ & 0.89 & $(0.53-1.50)$ \\
\hline \multicolumn{7}{|l|}{ Cancer fatalism } \\
\hline It seems like everything causes cancer & 0.85 & $(0.32-2.24)$ & 1.14 & $(0.64-2.03)$ & 1.23 & $(0.63-2.41)$ \\
\hline There's not much to do to lower cancer chances & 1.20 & $(0.45-3.22)$ & 1.21 & $(0.62-2.35)$ & 0.95 & $(0.42-2.13)$ \\
\hline Hard to know which cancer prevention recommendations to follow & 0.98 & $(0.31-3.14)$ & 1.92 & $(0.98-3.75)$ & 1.71 & $(0.73-4.03)$ \\
\hline \multicolumn{7}{|l|}{ Number of barriers to screening ${ }^{1}$} \\
\hline Cervical cancer & 0.51 & $(0.23-1.11)$ & - & - & 0.82 & $(0.47-1.42)$ \\
\hline Colorectal cancer & - & - & 1.18 & $(0.78-1.77)$ & 1.04 & $(0.68-1.59)$ \\
\hline \multicolumn{7}{|l|}{ Environmental factors } \\
\hline Travel time to doctor's office & 0.98 & $(0.93-1.04)$ & 1.01 & $(0.98-1.05)$ & 1.00 & $(0.96-1.04)$ \\
\hline Community social cohesion & 0.32 & $(0.06-1.79)$ & 0.60 & $(0.25-1.46)$ & 0.70 & $(0.21-2.36)$ \\
\hline
\end{tabular}

Note. Models adjusted for household income $(<\$ 50,000$ or $\$ 50,000$ or more), insurance status (private or other), and check-up in the last year (no or yes). Models assessing cervical cancer screening also adjusted for history of hysterectomy (no or yes)

${ }^{1}$ Barriers for screening for each cancer included don't know how to; don't need to; embarrassed; afraid; cost is too high; doctor is too far away (Supplementary Table S1) 
Fig. 1 Interactions between travel time from home to a primary care provider's office and cancer fatalism (it seems like everything causes cancer) in their relationship with (A) cervical cancer screening (interaction $p=.10)$ and (B) screening for both cervical and colorectal cancer (interaction $p=.01$ ) among women (ages 50-65) in rural, segregated counties of Pennsylvania, 2019-2020 $(n=100)$

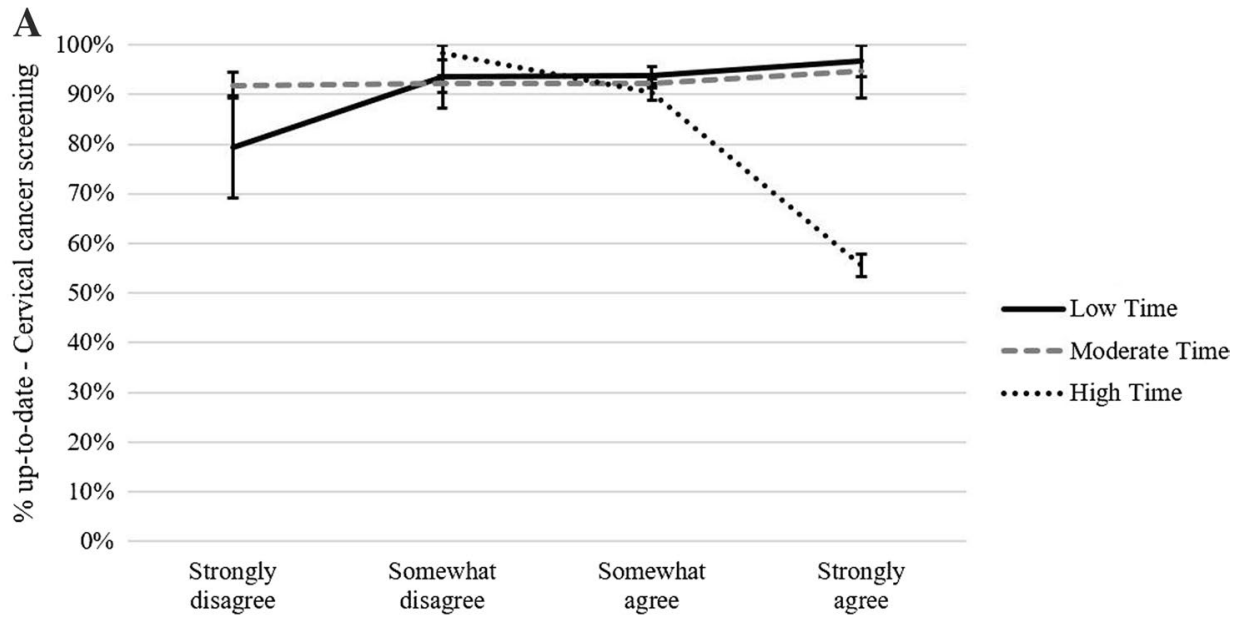

It seems like everything causes cancer

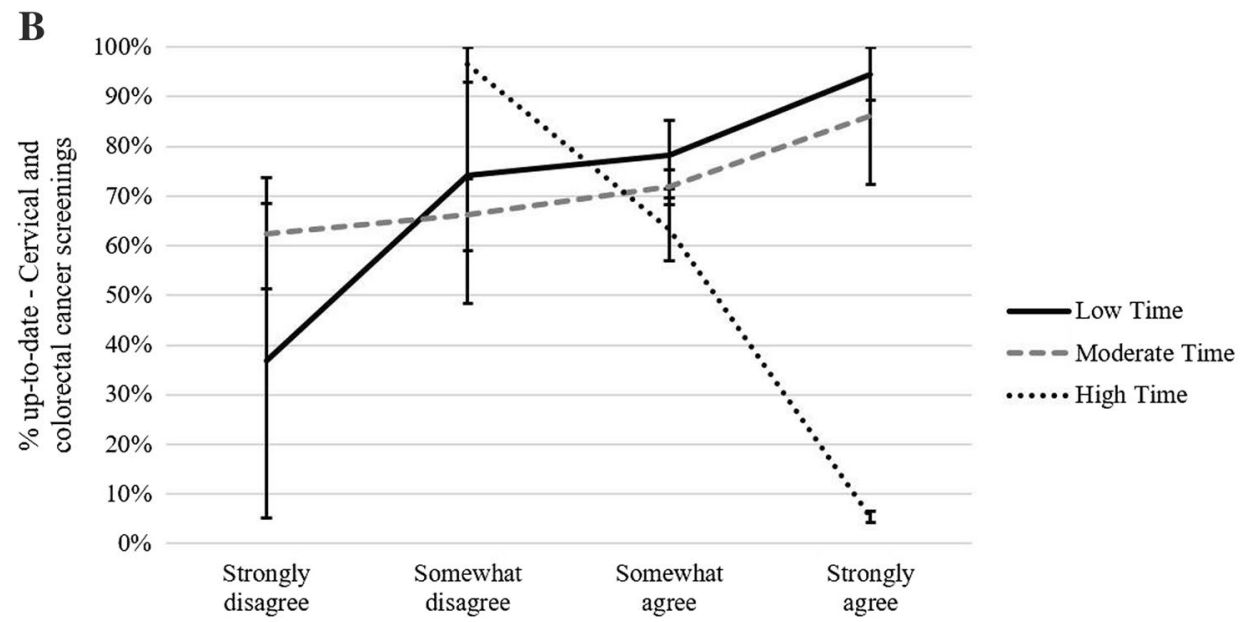

It seems like everything causes cancer interaction $p=0.02$, Fig. 2A; colorectal: interaction $p=0.05$, Fig. 2B; both cancers: interaction $p=0.02$, Fig. 2C). As an illustrative comparison, among participants without private insurance, uptake of cervical cancer screening decreased from $93 \%$ among participants who somewhat disagreed that patients should follow medical advice to $64 \%$ among participants who strongly agreed; participants with private insurance demonstrated the opposite relationship between healthcare trust and screening (Fig. 2A).

\section{Findings from Qualitative Focus Group Phase}

On average, participants were 58.8 years old $(S E=1.2)$ (Table 1). Most participants (73.3\%) lived in households with an annual income of $\$ 50,000$ or more, and most had private insurance $(75.0 \%)$ and had had a check-up in the last year $(81.3 \%)$.
Focus group discussions highlighted the compounding barriers these women faced in accessing care (Fig. 3) that spanned personal and environmental concerns. Below, we describe three themes about barriers to screening that emerged from the focus groups.

Barrier 1: Privacy Focus group participants raised the issue of privacy, which functioned both as a personal characteristic and as a response to healthcare situations. For example, one participant said, "A lot of people are private, and they don't like to go out to the doctors," reflecting the idea that preference for privacy, as an enduring personal characteristic, may make some people reluctant to seek care. Another participant said, "I take my privacy very private... even though it's only maybe you, the doctor, and a nurse, it's like you're out there for everybody to see," specifically in reference to cervical cancer screening, reflecting the idea that the lack of privacy inherent in certain clinical procedures is a deterrent from screening. 
Fig. 2 Interactions between insurance status and healthcare trust (patients should follow medical advice) in their relationship with (A) cervical cancer screening (interaction $p=.02),(\mathbf{B})$ colorectal cancer screening (interaction $p=.05$ ), and $(\mathbf{C})$ screening for both cancers (interaction $p=.02$ ) among women (ages 50-65) in rural, segregated counties of Pennsylvania, 2019-2020 $(n=100)$
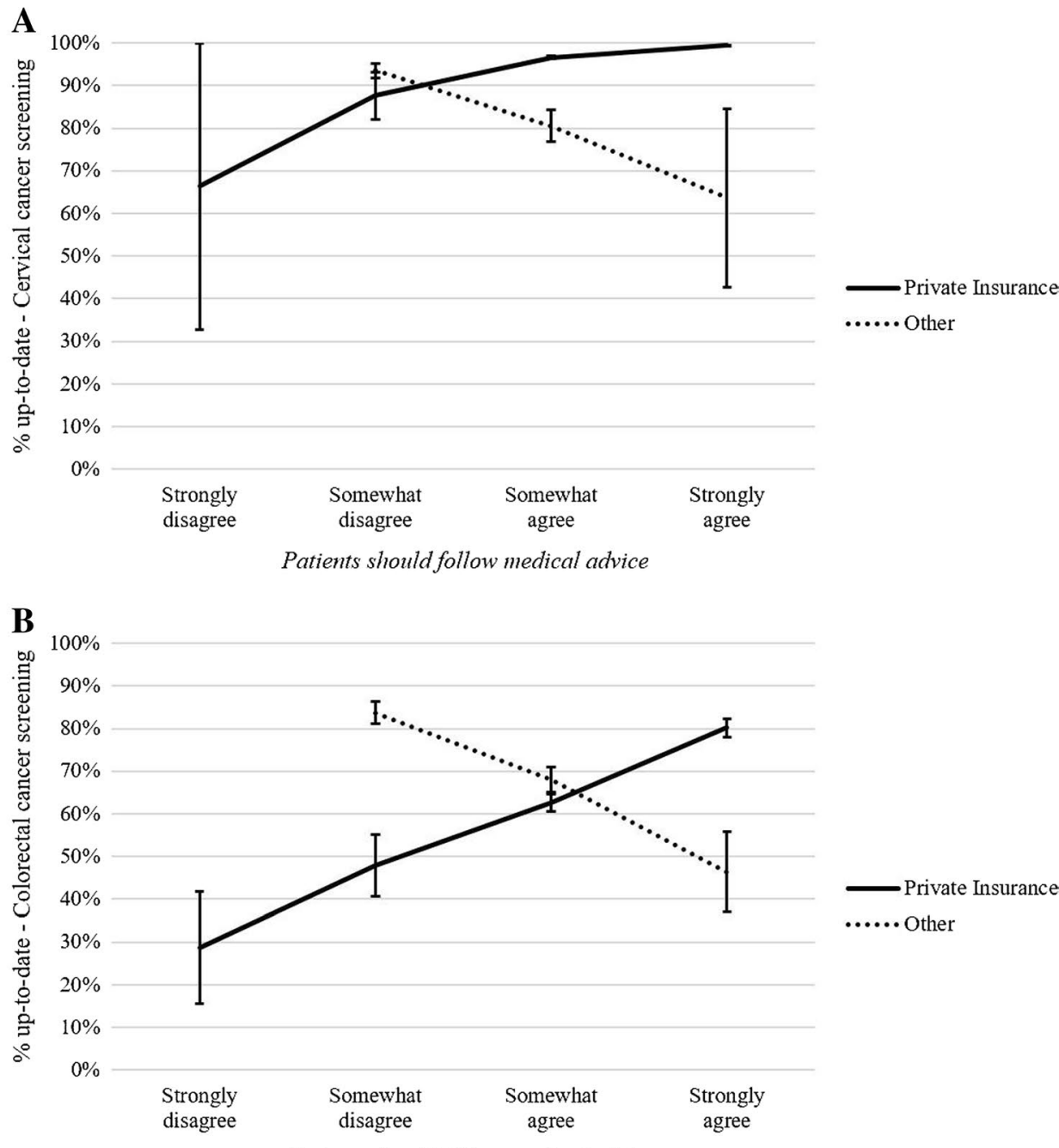

Patients should follow medical advice

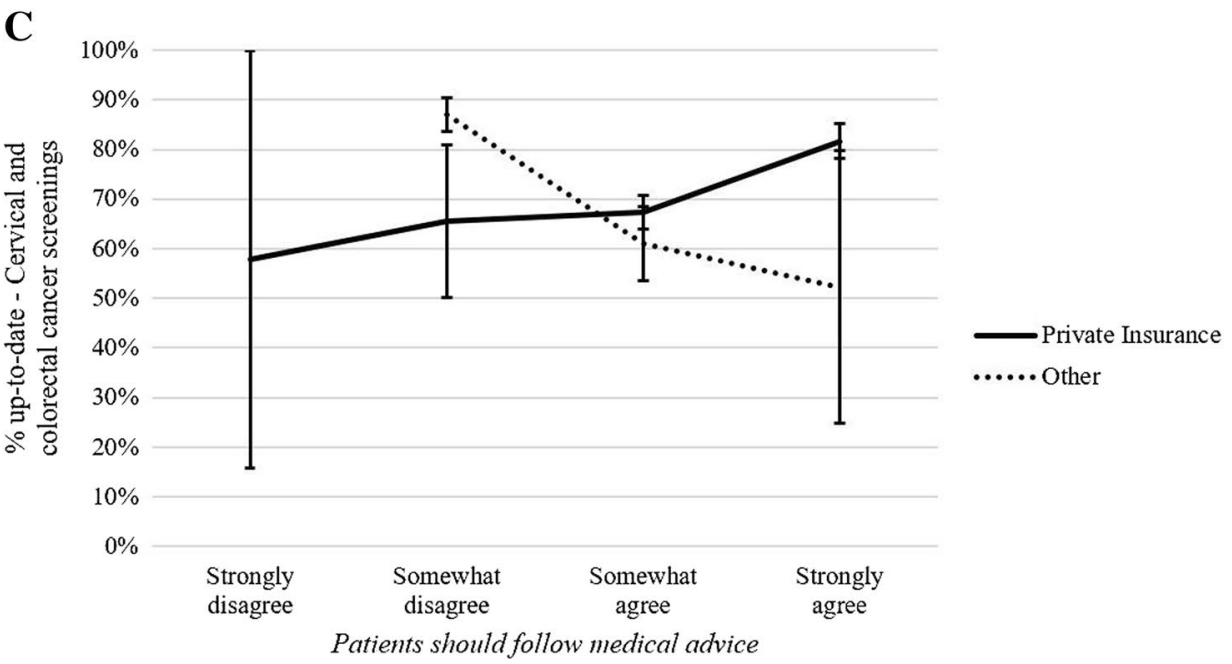

Barrier 2: Logistics When participants discussed the logistical requirements of cancer screening, they often described multiple multilevel barriers that, when combined, seemed insurmountable. For example, several participants discussed the exceptionally low availability of healthcare providers in their communities, which created difficulties in terms of time devoted to traveling to access screening. One participant reported driving up to $4 \mathrm{~h}$ to access specialized care. 


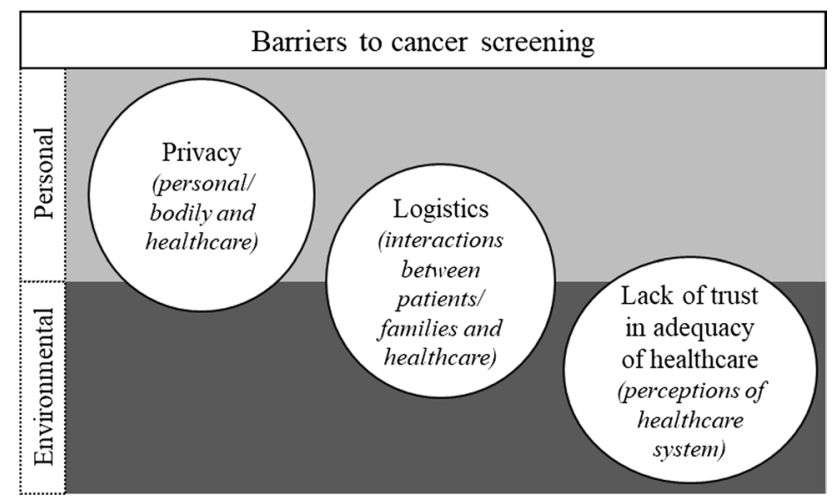

Fig. 3 Qualitative themes about barriers to cancer screening among women (ages 50-65) in rural, segregated counties of Pennsylvania, 2019-2020 $(n=16)$. All three barriers spanned personal and environmental domains

This travel burden impacted not only the participants, but also their family members; one participant said, "For someone else it's kind of inconvenient, but... you need the ride. So like for me, I'm a widow. I don't have a spouse, so I have to get somebody. Either my daughter has to take a day off, or someone [else] has to come with me." Some participants indicated that they did not like to drive into the city by themselves, or they had other conditions that made solo travel impractical. The time required for seeking care was especially burdensome for colonoscopies, which participants noted required "two or three days knocked right out of your week," as well as the accompaniment of a travel partner. Thus, logistical concerns for accessing care in this population included locating a provider, time and effort to travel to a clinic, and finding a companion or caregiver to accompany them.

These logistical concerns were compounded by issues of cost. Many participants were unsure of what the out-of-pocket costs could be for getting screening, and this uncertainty influenced their healthcare-seeking behaviors. For example, one participant said, "If you're having to pay out-of-pocket ... you have to ask the question, and you might be making choices based on your ability to pay." Several participants noted that travel time translated into time off of work, which could result in missed income.

Another major logistical barrier involved interacting with the local healthcare system, which many participants perceived as dysfunctional and inadequate (see the "Barrier 3: Lack of Trust in Adequacy of Healthcare" section below). They described their lengthy phone conversations and detailed record-keeping to facilitate communication and coordination among different providers, clinics, and insurance companies. Participants also expressed concerns that their local healthcare system was not well-equipped for providing care, referring patients to more distant facilities which necessitate even more logistical coordination. One participant said,
...If you do come out with a more advanced diagnosis, they ship you out immediately. No one in my area wants to deal with you. They want to send you to [a larger city]. They don't want to deal with it locally. They just want to send you away, and it can be frustrating... You don't necessarily have the time where you want to travel three, four hours for your treatment, but that's just how it's handled in my area.

Barrier 3: Lack of Trust in Adequacy of Healthcare Many focus group participants discussed their lack of trust in healthcare providers and the healthcare system as a barrier to cancer screening, a concept closely related to their perceptions of the availability of providers in their community. Some participants made succinct statements about their relationships with providers, such as "I' $m$ not one for doctors" or "I just don't like going to the doctor's office, and I tend to put it off until I absolutely have no choice [but] to go." Others described disappointing experiences seeking care or interacting with the healthcare system that eroded their trust. For example, one woman shared her experience of having a colonoscopy, which she verified with her insurance company would incur no out-of-pocket expense; however, she later received a large bill. She said, "Then they start playing the game with you... I learned my lesson. I have no desire to fork out another $\$ 350 \ldots$ for another colonoscopy."

Participants reflected that they could not rely on the healthcare system (including providers, clinics, and insurance companies) to operate in their best interest, or that the healthcare resources they had access to were low-quality. Several participants noted that older or less educated patients may be more likely to follow providers' recommendations unquestioningly, but in general, they regarded this as an unwise practice. One participant said, "Sadly, if you don't know enough to advocate for yourself, you die."

The combination of all of these personal and environmental logistical barriers was often overwhelming, especially in light of concerns about a cancer diagnosis. One participant said, "I've had a pap done, and then something came back that didn't look right. Then it was another test that needed to be done, retesting or going to a specialist at that point, so it's more days off work, more time off work, more expense, and then the worry that goes along with it. In my area, they're trying to get better, but you never get right in to see a specialist."

\section{Discussion}

In this mixed methods study of women from counties that are both rural and racially segregated, we found evidence for complex, multilevel relationships associated with cervical and colorectal cancer screening. Overall, screening 
was relatively high in this sample: $88.9 \%$ and $65.0 \%$ of participants reported being up-to-date with cervical and colorectal cancer screening, respectively, compared to $78 \%$ and $72 \%$ of eligible Pennsylvanian participants in the 2018 Behavioral Risk Factor Surveillance System survey [30]. However, the analysis identified barriers to screening that could serve as potential intervention targets in future studies. In the quantitative data, while single barriers to screening were not generally associated with being up-to-date with screening, interactions between access factors and personal barriers illustrated the deleterious effects that compounding barriers may have on cancer screening. The qualitative data revealed rich insights into decision-making around cancer screening, including privacy concerns, logistical barriers, and lack of trust in the adequacy of local healthcare services. Ultimately, the findings did not provide support for our hypothesis that being up-to-date with screening would be lower among women reporting more barriers to screening, but taken together, the quantitative and qualitative analyses demonstrated that several multilevel barriers may operate interactively to influence uptake of cancer screening.

Associations between geographic location and cancer mortality are relatively well-known [8,31]. Incidence and mortality rates for cancers with evidence-based screening modalities (including cervical and colorectal cancers) are higher [7, 8, 32], and participation in screening is lower [4, 5] for rural versus urban populations. Differences in screening by segregation are less clear [6], and in fact, empirical and theoretical work suggests that segregation may offer some health benefits to residents [33]. Often, geographic differences in health outcomes are attributed to local variation in other community factors (e.g., poverty [32]) or healthcare system factors (e.g., access to quality care [34]). Our study findings support these proposed mechanisms. For example, survey participants reported that cost was a barrier to screening, which was associated with lower odds of being up-to-date, indicating that finances are still important considerations for this population. In addition, focus group participants reported concerns over the quality of care provided by their local health systems and were often referred to specialists in (distant) metropolitan areas. Notably, all 14 rural, segregated counties in the current study are also designated as Health Professional Shortage Areas [35], reflecting the challenges rural patients face in accessing care locally and potentially explaining participants' perceptions of lowquality care. The qualitative phase of the study also revealed at least two barriers to screening that we did not assess in the quantitative phase that may be especially pertinent for rural, segregated, or other underserved populations. First, participants reported their feelings of privacy that made cancer screening uncomfortable [36]. Second, participants expressed their reluctance to be a burden on their family members by asking them for assistance in accessing care, particularly for colorectal cancer screening. Overall, these findings support previous empirical and theoretical work that suggests multilevel factors may give rise to health disparities [17].

The interactions among barriers to screening were observed in both the quantitative and qualitative analyses. Quantitatively, measures of access to care (i.e., travel time, insurance status) and intrapersonal attitudes (i.e., fatalism, trust) interacted such that the combination of these barriers were uniquely associated with lower probability of getting screened. Thus, it appears that participants are able to address and overcome individual barriers to care, but the accrual of multiple, multilevel, interacting barriers was more difficult to overcome. It is unclear how compounding barriers may have affected screening behaviors-whether they were associated with (1) lower intention or motivation to get screened and/or (2) reduced ability to translate intentions/ motivation into behavior-but future studies should examine these issues further. Importantly, these findings should be considered preliminary, since our analysis was not powered to fully parse all the potential interactions among access and attitudinal variables. Qualitatively, participants discussed how privacy, logistics, and perceptions of the adequacy of healthcare services, together, created an environment that was not supportive of cancer screening. Logistical concerns related to travel and cost/insurance barriers existed in a liminal space with concerns about adequacy and quality of healthcare services: Participants perceived a trade-off between the more local services that were of lower quality versus more distant services (i.e., in metropolitan areas) that were of higher quality. Rural communities tend to have lower access to preventive and specialty healthcare services [37, 38 ], which could result in lower continuity and quality of care. This perception of inadequate care could be related to participants' experiences having to "advocate" for themselves within the healthcare/insurance systems, which can be unnecessarily difficult and confusing. Indeed, self-advocacy was discussed as a method to overcome barriers to care, but focus group participants often acknowledged that engaging in these activities could be less feasible or effective for other people in their communities (e.g., women who are poorer or have jobs with inflexible schedules). One potential method to overcome the logistical and trust barriers reported by participants in this study is the use of self-sampling modalities for colorectal cancer screening (i.e., stool testing [3]) and cervical cancer screening (i.e., self-collected HPV testing, which is an emerging technology), which may be especially impactful for underserved patients [39], including those living in rural and racially segregated populations.

This study had several strengths and limitations. In terms of strengths, this study was successful in providing insights into a poorly understood population of women living in rural, segregated (i.e., predominantly non-Hispanic white) 
communities. Additionally, we were able to assess exploratory cross-level interactions between multilevel factors related to cancer screening, allowing us to better understand the context of cancer screening decision-making. Another strength is in the mixed methods study design; the qualitative findings provided rich contextual details on the quantitative findings, providing a better understanding of attitudes and behaviors related to cancer screening. Lastly, we were able to study multiple health behaviors (i.e., screening for two types of cancer) that are relevant to this study population in one efficient study design. In terms of limitations, we used a convenience sample for the cross-sectional survey. While the survey and focus group samples were relatively representative of the population of the eligible counties, participants had higher levels of education, which could influence some of the findings. Thus, the results may not generalize all communities or other rural, segregated communities. Relatedly, the small sample size precludes detailed statistical analysis of study variables. Second, all variables were self-reported and may not be completely reliable. We also cannot make any conclusions regarding temporality due to the cross-sectional study design. We did not include breast cancer screening as a study outcome as this is not routinely available in the primary care setting. Other guidelines for cancer screening exist, but we chose to focus on those provided by the USPSTF. Notably, we did not capture receipt of colonoscopy in the last 6-10 years, which likely results in an underestimation of the proportion of participants up-todate with colorectal cancer screening. Data collection coincided with the beginning of the COVID-19 pandemic, which could have impacted recruitment and responses to survey and focus group questions. This issue is most pertinent for the focus groups, since data collection had to take place virtually using a teleconferencing platform. Finally, we chose county as the unit of observation for both rural and segregated designations. This may mask small area differences that are missed at this higher level, potentially introducing unknown heterogeneity. In Pennsylvania, racially segregated counties have predominantly non-Hispanic white populations; findings may not apply to racially segregated counties with different racial/ethnic compositions.

This exploratory study used mixed methods to conduct in-depth analysis of the lived experiences of women living in rural, racially segregated communities to understand barriers to cancer screening. Our findings indicate that, individually, most barriers are not closely related to uptake of screening, but in combination, multilevel barriers are associated with marked differences in uptake. Future studies should examine these patterns in different communities and use these results to inform interventions to promote cancer screening. Given the multilevel nature of these barriers, multilevel interventions are needed [16, 17]. A promising intervention that could be implemented in coordination with primary care is self-sampling for cancer screening [40, 41], which overcomes certain individual and environmental barriers to screening, e.g., those related to privacy, logistics, and quality of care.

Supplementary Information The online version contains supplementary material available at https://doi.org/10.1007/s13187-021-02069-0.

Funding This study was funded by K22 CA225705 (PI: Moss, J. L.).

\section{References}

1. American Cancer Society (2020) Cancer Facts \& Figures 2020. Available from: https://www.cancer.org/content/dam/cancer-org/ research/cancer-facts-and-statistics/annual-cancer-facts-and-figur es/2020/cancer-facts-and-figures-2020.pdf. Accessed 25 June 2021

2. US Preventive Services Task Force (2018) Final recommendation statement: cervical cancer: Screening. Available from: https:// www.uspreventiveservicestaskforce.org/Page/Document/Recom mendationStatementFinal/cervical-cancer-screening2. Accessed 25 June 2021

3. U. S. Preventive Services Task Force (2021) Final recommendation statement: Colorectal cancer: Screening. Available from: https://www.uspreventiveservicestaskforce.org/uspstf/recom mendation/colorectal-cancer-screening. Accessed 25 June 2021

4. Anderson AE, Henry KA, Samadder NJ, Merrill RM, Kinney AY (2013) Rural vs urban residence affects risk-appropriate colorectal cancer screening. Clin Gastroenterol Hepatol 11(5):526-533

5. Coughlin SS, Thompson TD, Hall HI, Logan P, Uhler RJ (2002) Breast and cervical carcinoma screening practices among women in rural and nonrural areas of the United States, 1998-1999. Cancer 94(11):2801-2812

6. Moss JL, Ehrenkranz R, Perez LG, Hair BY, Julian AK (2019) Geographic disparities in cancer screening and fatalism among a nationally representative sample of US adults. J Epidemiol Community Health 73(12):1128-1135

7. Blake KD, Moss JL, Gaysynsky A, Srinivasan S, Croyle RT (2017) Making the case for investment in rural cancer control: an analysis of rural cancer incidence, mortality, and funding trends. Cancer Epidemiol Biomark Prev 26(7):992-997

8. Henley SJ, Anderson RN, Thomas CC, Massetti GM, Peaker B, Richardson LC (2017) Invasive cancer incidence, 2004-2013, and deaths, 2006-2015, in nonmetropolitan and metropolitan counties - United States. Morb Mortal Wkly Rep Surveill Summ (Washington, DC : 2002) 66(14):1-13

9. Figueroa JB, Breen N (1995) Significance of underclass residence on the stage of breast or cervical cancer diagnosis. Am Econ Rev 85(2):112-116

10. Reyes-Ortiz CA, Eschbach K, Zhang DD, Goodwin JS (2008) Neighborhood composition and cancer among Hispanics: tumor stage and size at time of diagnosis. Cancer Epidemiol Biomark Prev 17(11):2931-2936

11. Hall KL, Oh A, Perez LG, Rice EL, Patel M, Czajkowski S et al (2018) The ecology of multilevel intervention research. Transl Behav Med 8(6):968-978

12. Gaskin DJ, Dinwiddie GY, Chan KS, McCleary R (2012) Residential segregation and disparities in health care services utilization. Med Care Res Rev 69(2):158-175

13. Befort CA, Nazir N, Engelman K, Choi W (2013) Fatalistic cancer beliefs and information sources among rural and urban adults in the USA. J Cancer Educ 28(3):521-526 
14. Segel JE, Lengerich EJ (2020) Rural-urban differences in the association between individual, facility, and clinical characteristics and travel time for cancer treatment. BMC Public Health 20(1):196

15 Massey DS (1996) The age of extremes: concentrated affluence and poverty in the twenty-first century. Demography. 33(4):395412 (discussion 3-6)

16. Bandura A (2011) Social cognitive theory. Handbook of Social Psychological Theories. 349-73

17. Warnecke RB, Oh A, Breen N, Gehlert S, Paskett E, Tucker $\mathrm{KL}$ et al (2008) Approaching health disparities from a population perspective: the National Institutes of Health Centers for Population Health and Health Disparities. Am J Public Health 98(9):1608-1615

18. U. S. Department of Agriculture (2019) Rural-urban continuum codes: overview. Available from: http://www.ers.usda.gov/dataproducts/rural-urban-continuum-codes.aspx. Accessed 25 June 2021

19. Landrine H, Corral I (2014) Advancing research on racial-ethnic health disparities: improving measurement equivalence in studies with diverse samples. Front Public Health 2:282

20. U. S. Census Bureau (2014) American FactFinder. Available from: http://factfinder2.census.gov/faces/nav/jsf/pages/index.xhtml. Accessed 25 June 2021

21. U. S. Census Bureau (2018) American Community Survey (ACS). Available from: https://www.census.gov/programs-surveys/acs/. Accessed 25 June 2021

22. Turgeon M, Havard M, Pallera H, Diller P, Gefter L. Levels of trust in the healthcare system among low-income youth: an evaluation of an assessment tool for a school-based health promotion program

23. National Cancer Institute (2015) Health information national trends survey 4 (HINTS 4): Cycle 4 methodology report. Available from: https://hints.cancer.gov/docs/Instruments/HINTS_4_ Cycle_4_Methodology_Report_Final.pdf. Accessed 25 June 2021

24. Janz NK, Wren PA, Schottenfeld D, Guire KE (2003) Colorectal cancer screening attitudes and behavior: a population-based study. Prev Med 37(6):627-634

25. O'Brien DJ, McClendon MJ, Ahmed A (1989) Neighborhood community and quality of life. Community Dev 20(2):59-71

26. Kerr C, Nixon A, Wild D (2010) Assessing and demonstrating data saturation in qualitative inquiry supporting patient-reported outcomes research. Expert Rev Pharmacoecon Outcomes Res 10(3):269-281

27. Bradley EH, Curry LA, Devers KJ (2007) Qualitative data analysis for health services research: developing taxonomy, themes, and theory. Health Serv Res 42(4): 1758-1772

28. Elo S, Kyngäs H (2008) The qualitative content analysis process. J Adv Nurs 62(1):107-115

29. Tausch AP, Menold N (2016) Methodological aspects of focus groups in health research: results of qualitative interviews with focus group moderators. Glob Qual Nurs Res $3: 2333393616630466$
30. Centers for Disease Control and Prevention (2019) Behavioral risk factor surveillance system. Available from: http://www.cdc.gov/ brfss/. Accessed 25 June 2021

31. Mokdad AH, Dwyer-Lindgren L, Fitzmaurice C, Stubbs RW, Bertozzi-Villa A, Morozoff C et al (2017) Trends and patterns of disparities in cancer mortality among US counties, 1980-2014. JAMA 317(4):388-406

32. Zahnd WE, James AS, Jenkins WD, Izadi SR, Fogleman AJ, Steward DE et al (2018) Rural-urban differences in cancer incidence and trends in the United States. Cancer Epidemiol Biomark Prev 27(11):1265-1274

33 White K, Haas JS, Williams DR (2012) Elucidating the role of place in health care disparities: the example of racial/ethnic residential segregation. Health Services Research. 47(3pt2):1278-99

34. Charlton M, Schlichting J, Chioreso C, Ward M, Vikas P (2015) Challenges of rural cancer care in the United States. Oncology (Williston Park) 29(9):633-640

35. U.S. Health Resources \& Services Administration (2020) Health professional shortage areas (HPSAs). Available from: https://bhw. hrsa.gov/shortage-designation/hpsas. Accessed 25 June 2021

36. Wang H, Roy S, Kim J, Farazi PA, Siahpush M, Su D (2019) Barriers of colorectal cancer screening in rural USA: a systematic review. Rural Remote Health 19(3):5181

37. Belasco EJ, Gong G, Pence B, Wilkes E (2014) The impact of rural health care accessibility on cancer-related behaviors and outcomes. Appl Health Econ Health Policy 12(4):461-470

38. Moss JL, Liu B, Feuer EJ (2017) Urban/rural differences in breast and cervical cancer incidence: the mediating roles of socioeconomic status and provider density. Womens Health Issues 27(6):683-691

39. Katz ML, Zimmermann BJ, Moore D, Paskett ED, Reiter PL (2016) Perspectives from health-care providers and women about completing human papillomavirus (HPV) self-testing at home. Women Health 1-17

40. Vanderpool RC, Jones MG, Stradtman LR, Smith JS, Crosby RA (2014) Self-collecting a cervico-vaginal specimen for cervical cancer screening: an exploratory study of acceptability among medically underserved women in rural Appalachia. Gynecol Oncol 132(Suppl 1):S21-S25

41. Briant KJ, Sanchez JI, Ibarra G, Escareño M, Gonzalez NE, Jimenez Gonzalez V et al (2018) Using a culturally tailored intervention to increase colorectal cancer knowledge and screening among Hispanics in a rural community. Cancer Epidemiol Biomark Prev 27(11):1283-1288

Publisher's Note Springer Nature remains neutral with regard to jurisdictional claims in published maps and institutional affiliations. 